\title{
Education and screening for chronic kidney disease in Indian youth: pilot program results
}

\author{
Panduranga S Rao' \\ Julie A Wright Nunes' \\ Brenda W Gillespie ${ }^{2}$ \\ Rachel L Perlman' \\ Rajan Ravichandran ${ }^{3}$ \\ 'Division of Nephrology, Department \\ of Internal Medicine, University of \\ Michigan Health System, ${ }^{2}$ Department \\ of Biostatistics, School of Public \\ Health, University of Michigan, Ann \\ Arbor, MI, USA; ${ }^{3}$ Department of \\ Nephrology, Madras Institute of \\ Nephrology, Madras Institute of \\ Orthopedics and Trauma Hospitals, \\ Chennai, India
}

Correspondence: Julie A Wright Nunes Division of Nephrology, Department of Internal Medicine, University of Michigan Health System, 102 Observatory Road, Simpson Memorial Building, Ann Arbor, MI 48I09, USA

Tel +l 7346153994

Fax +I 7346154887

Email juwright@med.umich.edu
This article was published in the following Dove Press journal: International Journal of Nephrology and Renovascular Disease 24 April 2017

Number of times this article has been viewed

Background: There is a paucity of information on kidney education and screening programs in Indian youth.

Methods: Participants $(n=2,158)$ from Chennai colleges were educated about the kidneys and chronic kidney disease (CKD) and screened in a pilot program from April to May 2013. This entailed: 1) a presentation and educational video and 2) an on-site assessment of weight, blood pressure, and demographic information. Urinalysis (UA) kits were distributed and returned in $\leq 48$ hours. We examined participant characteristics and their association with dipstick proteinuria using logistic regression.

Results: The mean (standard deviation [SD]) age was 18.9 (1.6) years, and 1,451 (68\%) were men. Mean (SD) body mass index (BMI) was $21.9(4.3) \mathrm{kg} / \mathrm{m}^{2} ; 745(36 \%)$ had a BMI consistent with being overweight or obese. Mean (SD) systolic blood pressure (SBP) was 118.7 (13.1) $\mathrm{mm} \mathrm{Hg}$, and $94(5 \%)$ of the participants had SBP $\geq 140$. Mean (SD) diastolic blood pressure (DBP) was 70.9 (11.4) $\mathrm{mm} \mathrm{Hg}$, with 119 participants (6\%) having $\geq 90 \mathrm{~mm} \mathrm{Hg}$. A total of 136 participants had glycosuria $(\mathrm{UA} \geq 1+)$ and $120(6 \%)$ had proteinuria $(\mathrm{UA} \geq 1+)$. In unadjusted analyses, sex (odds ratio $[\mathrm{OR}]=1.64$ [confidence interval, CI 1.06-2.55]; $p=0.026$ men vs. women) and age $(\mathrm{OR}=1.13$ per year [CI $1.01-1.26] ; p=0.032)$ were significantly associated with proteinuria. In the analysis adjusted for age, sex, SBP, DBP, glycosuria, and BMI, age remained independently associated with higher odds for proteinuria $(\mathrm{OR}=1.14$ per year [1.02-1.29]; $p=0.026)$. Males showed a trend of higher risk compared with women ( $\mathrm{OR}=1.57$ [CI 1.00-2.50]; $p=0.051)$.

Conclusion: This education and screening pilot program in a population of college students offers unique opportunities for identification, education, and early intervention for CKD.

Keywords: screening, youth, CKD, proteinuria, glycosuria, education, hypertension, BMI, Indian youth

\section{Background}

The prevalence of risk factors for chronic kidney disease (CKD) in Indian adults is high. In the year 2000, there were an estimated 31 million people with diabetes in India; projections indicate that this will increase to 79 million by the year $2030 .{ }^{1}$ Similarly, hypertension is becoming increasingly common, with a recent study reporting prevalence as high as $20 \% .^{2}$ Diabetes and hypertension together are responsible for $75 \%$ of renal failure in developed countries. ${ }^{3}$ Thus, the increasing number of people affected by these conditions in India is concerning for a future epidemic of renal failure within its population.

Although some debate exists about how to most effectively identify and educate persons with CKD in general populations, ${ }^{4}$ research shows that early identification 
and intervention can provide great benefit. Efforts in the US through the Kidney Early Evaluation Program (KEEP) focused on early screening and education to people with CKD and risk factors. Follow-up showed that participants in KEEP were more likely to seek and receive renal care and experience less mortality and morbidity compared with nonparticipants. ${ }^{5}$

Screening efforts have been reported in India, but there is little information available about programs combining screening for risk factors along with education in younger vulnerable groups. Prior research has focused on obtaining prevalence estimates of CKD in adults. Most of these studies have been published over the past decade. Methods and definitions across studies vary widely. Mani ${ }^{6}$ reported a CKD prevalence of $0.16 \%$ in rural south India in a population of 25,000. Agarwal et $\mathrm{al}^{7}$ performed a study in 4,972 patients in urban New Delhi and found CKD in $0.79 \%$. Contrary to earlier reports, a later study using a population of 6,120 patients in medical centers showed a much higher prevalence of $17 \%{ }^{8}$

The genesis of kidney disease likely starts early in youth and largely with the onset of modifiable risk factors. ${ }^{9}$ Early screening to identify these risk factors, combined with education about how to keep kidneys healthy, could provide great opportunity to prevent or abate kidney disease before it becomes a problem. Here, we report the results of a pilot program designed to screen Indian youth for CKD risk factors and educate them about the kidney and health behaviors necessary to preserve renal function. The program was implemented as a "grass roots" effort. It was inspired and driven by local community leaders, who were highly motivated to serve their public and curb the growing epidemic of chronic disease within India.

\section{Methods}

The pilot program was initially designed by a nephrologist (RR) and a team of support personnel, primarily as an educational outreach. The team of support personnel had a health background and were trained to check height and weight and measure blood pressure. Along with delivery of an education module, pilot testing for screening was performed for CKD risk factors. Overall goals of the program were to 1) educate young adults about the kidneys and how to keep them healthy and 2) assess the ability to efficiently perform one-time screening for CKD risk factors. The project was initially launched and completed as a grass roots effort to increase awareness of kidney disease. Given the alarming findings, it was felt that it should be disseminated in the scientific community by publishing the findings and presenting it at meetings. Hence, it was presented to the ethics board at Madras Institute of Orthopedics and Trauma (MIOT), Chennai, India, and the Institutional Review Board at the University of Michigan who approved the analysis of deidentified data.

The program was delivered to students enrolled in one of five colleges throughout Chennai, India, from April through May 2013. The program was advertised through posters and flyers distributed in the college. The educational portion entailed a 10-minute presentation and video on topics related to CKD. These included the asymptomatic nature of CKD and urine testing to detect kidney disease. The video portion was also made available on YouTube for a period of time thereafter, to allow students to access the information again later. After the brief educational session, trained personnel collected information on volunteers, including age, sex, height, weight, and blood pressure. Information about preexisting diabetes and hypertension was not specifically sought. There were no exclusion criteria. Blood pressure was taken after participants were seated for at least 10 minutes and was recorded in the sitting position with the right arm supported at heart level using an automated blood pressure cuff (Citizen Model CH-432).

For the urine screening portion of the program, specific information was first provided on urine collection techniques. Urine analysis kits consisting of a urine dipstick and container with a color-coded guide to interpret results were distributed to all students at the time of education and assessment (Figure 1). No instruction about early morning sample of urine was given to the students. If the urine sample was not returned during the screening, the students returned the sample to the study personnel within 48 hours.

For analysis, hypertension was defined as either a systolic blood pressure (SBP) $\geq 140 \mathrm{~mm} \mathrm{Hg}$ or a diastolic blood pressure (DBP) $\geq 90 \mathrm{~mm} \mathrm{Hg}$. Body mass index (BMI) was defined as weight in kilograms divided by the square of height in meters. Overweight was defined as BMI greater than 23 and obese as greater than 25 per consensus statements appropriate to India. ${ }^{10}$ Urine tests were on a 4 point scale for glucose and protein and were deemed positive for any value $\geq 1+$.

Statistical analysis was done with STATA version 10.0 (College Station, TX, USA). In descriptive analysis, continuous variables are expressed as mean values with standard deviations (SDs) and categorical variables as n (\%). Logistic regression was used to test associations between proteinuria as the outcome and participant characteristics as independent predictors, with results presented as odds ratios (ORs). $p$ values $<0.05$ were considered significant. 


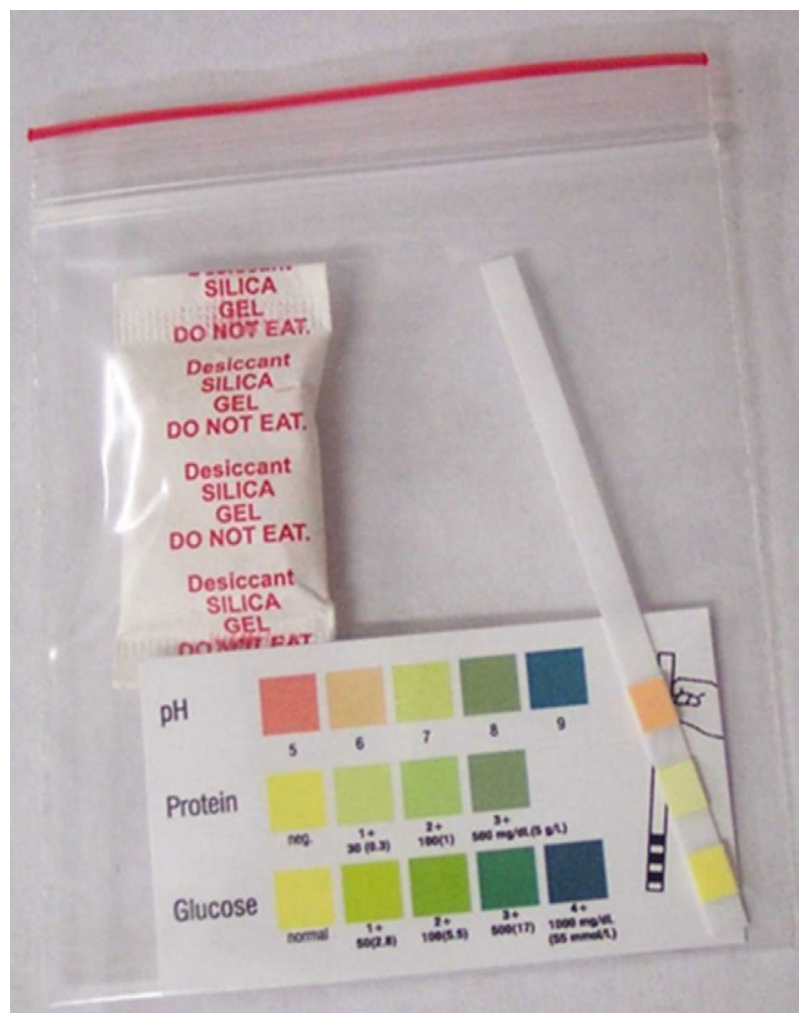

Figure I Urine dipstick testing packet and color-coded guide distributed to volunteers.

\section{Results}

A total of 2,158 students were enrolled from five colleges in Chennai. Baseline characteristics are shown in Table 1. The mean (SD) age was 18.92 (1.60) years, and 1,451 (68\%) were men. Measurement and urine analysis were completed for 2,035 (94\%) students. Mean (SD) BMI was 21.90 (4.29) kg/ $\mathrm{m}^{2}$, and $745(36 \%)$ had a BMI consistent with being overweight or obese $\left(\geq 23 \mathrm{~kg} / \mathrm{m}^{2}\right)$. Mean (SD) SBP was 118.71 (13.06) $\mathrm{mm} \mathrm{Hg}$, and 94 (5\%) of the participants had SBP $\geq 140$. Mean (SD) DBP was 70.92 (11.42) $\mathrm{mm} \mathrm{Hg}$, with 119 participants $(6 \%)$ having $\mathrm{DBP} \geq 90$. A total of 136 students had glycosuria (urinalysis $[\mathrm{UA}] \geq 1+$ ), and 120 (6\%) had abnormal urine protein $(\mathrm{UA} \geq 1+$ ).

In univariate analysis, male sex (OR 1.64 [confidence interval, CI 1.06-2.55]; $p=0.026$ men compared to women) and older age (OR 1.13 [CI 1.01-1.26]; $p=0.032$ each year older compared to younger) were significantly associated with proteinuria. In the analysis adjusted for age, sex, SBP, DBP, glycosuria, and BMI, age remained independently associated with higher odds for proteinuria (1.14 [1.02-1.29]; $p=0.026$ older compared to younger). Sex showed a trend toward significance (1.57 [CI 1.00-2.50]; $p=0.051$ for men compared with women) but did not meet the cutoff for significance (Table 2). Most students who viewed the video volunteered for urine screening, and a high percentage of them returned the UA test kits, 2,035 (94\%).

\section{Discussion}

This grass roots effort led by community leaders to promote a CKD education and screening program in youth was both feasible and revealing. The program provided kidney education to young adults efficiently, reaching $>2,000$ students within a 1-month period. Several studies over the past decade have attempted to infer the prevalence estimates of CKD in India. Methods and definitions of CKD across studies varied widely. Mani ${ }^{6}$ reported a CKD prevalence of $0.16 \%$ in rural south India in a population of 25,000. Agarwal et $\mathrm{al}^{7}$ performed a study in 4,972 patients in urban New Delhi and found CKD in $0.79 \%$. Contrary to earlier reports, a later study using a population of 6,120 patients in medical centers showed a much higher prevalence of $17 \%{ }^{8}$ To our knowledge, this is the first report on Indian youth describing current prevalence of CKD risk factors such as hypertension and proteinuria, along with an examination of associations between individual characteristics and proteinuria. The results provide evidence for concern related to the growing epidemic of kidney disease that can already be identified in young people. The program was novel in concept, motivated locally, and easy to administer. It also suggests that future efforts for primary prevention should be delivered before adulthood - where the majority of this type of work has been reported in the past. ${ }^{11,12}$

The idea of a youth-focused program to provide health education aimed at reducing chronic disease is not novel but is not well reported for CKD. Programs outside of CKD have been used to optimize self-care in reproductive and sexual health, ${ }^{13}$ reduce obesity, ${ }^{14}$ and lower student propensity for risky behaviors. ${ }^{15}$ A US program called "Project Healthy Schools" (PHS) has found success in reaching students even earlier, in middle school, educating them about behaviors aligned with good cardiovascular health. ${ }^{16}$ The PHS program was, however, not directed toward kidney health, and screening is not part of the PHS program. As a collaborative partnership between an academic medical institution, health payors, and schools, PHS focuses on education about health behaviors needed to feel good today, instead of campaigning for just doing these things to stay healthy for the future. The program couples short education modules with community partnerships to ensure PHS remains tailored to the needs of each school and can be sustained over time within individual school environments. Since PHS implementation, health benefits have been observed in students across participating 
Table I Baseline characteristics $(n=2,158)$

\begin{tabular}{|c|c|c|c|}
\hline Characteristics & Women,* mean (SD) or $\mathrm{n}(\%)$ & Men,* mean (SD) or n (\%) & All participants, mean (SD) or $n(\%)$ \\
\hline Sex & 707 (33\%) & $|45|(67 \%)$ & $2158(100 \%)$ \\
\hline Age, years & $18.5(1.7)$, range $15-26$ & 19.1 (I.5), range $15-25$ & $18.9(1.6)$, range $15-26$ \\
\hline Body mass index (BMI), $\mathrm{kg} / \mathrm{m}^{2}$ & $21.2(4.7)$, range $11.2-57.8$ & $22.3(4.0)$, range $|3.2-5| .0$ & $21.9(4.3)$, range $11.2-57.8$ \\
\hline Overweight or obese ${ }^{* *}$ & $194(30)$ & $551(38)$ & $745(36)$ \\
\hline Systolic blood pressure, $\mathrm{mm} \mathrm{Hg}$ & II 2.5 (12.9), range $8 \mid-173$ & $121.5(12.1)$, range $89-173$ & 118.7 (I3.I), range $8 I-173$ \\
\hline Diastolic blood pressure, $\mathrm{mm} \mathrm{Hg}$ & 70.1 (II.0), range $27-125$ & 71.3 (11.6), range $16-135$ & 70.9 (1I.4), range $16-135$ \\
\hline \multicolumn{4}{|l|}{ Urinalysis (UA)* } \\
\hline Glycosuria*** & $35(5)$ & I0I (7) & $136(6)$ \\
\hline Proteinuria*** & $27(4)$ & $93(6)$ & $120(6)$ \\
\hline
\end{tabular}

Notes: *Based on available data. **Defined as BMI $\geq 23 \mathrm{~kg} / \mathrm{m}^{2}$ per Indian consensus statement. ${ }^{10} * * * \mathrm{UA}$ dipstick value $\geq 1+$.

Abbreviations: SD, standard deviation; N/A, not applicable.

Table 2 Unadjusted and adjusted* ORs for proteinuria

\begin{tabular}{|c|c|c|}
\hline Characteristics & $\begin{array}{l}\text { Unadjusted OR }(\mathrm{Cl}) ; \\
\text { p value }\end{array}$ & $\begin{array}{l}\text { Adjusted OR }(\mathrm{Cl}) ; \\
\text { p value }\end{array}$ \\
\hline $\begin{array}{l}\text { Age, (per I year } \\
\text { increase) }\end{array}$ & $1.13(1.01-1.26) ; 0.032$ & I.I (I.02-1.29); 0.026 \\
\hline \multicolumn{3}{|l|}{ Sex } \\
\hline Female & Reference & Reference \\
\hline Male & 1.64 (I.06-2.55); 0.026 & 1.57 (I.00-2.50); $0.05 \mathrm{I}$ \\
\hline \multicolumn{3}{|l|}{ BMI, kg/m² } \\
\hline$<23$ & Reference & Reference \\
\hline$\geq 23$ & I.22 (0.84-I.80); 0.29 & I.IS (0.78-I.7I); 0.47 \\
\hline \multicolumn{3}{|l|}{ SBP, mm Hg } \\
\hline$<140$ & Reference & Reference \\
\hline$\geq 140$ & I.I 4 (0.49-2.67); 0.76 & 0.95 (0.39-2.32); 0.92 \\
\hline \multicolumn{3}{|l|}{ DBP, mm Hg } \\
\hline$<90$ & Reference & Reference \\
\hline$\geq 90$ & I.2I (0.58-2.55); $0.6 \mathrm{I}$ & I.07 (0.47, 2.42); 0.87 \\
\hline \multicolumn{3}{|l|}{ Glycosuria } \\
\hline$<1+$ & Reference & Reference \\
\hline$\geq 1+$ & I. 19 (0.59-2.40); 0.63 & 1.06 (0.50-2.23); 0.88 \\
\hline
\end{tabular}

Note: *Adjusted model included: age, sex, BMI, SBP, DBP, and glycosuria, with proteinuria as outcome variable.

Abbreviations: OR, odds ratio; $\mathrm{Cl}$, confidence interval; $\mathrm{BMI}$, body mass index; $\mathrm{SBP}$, systolic blood pressure; DBP, diastolic blood pressure.

schools, including significant decreases in serum cholesterol levels, serum blood glucose, and mean blood pressures - all correlated with increases in vegetable and fruit consumption, increases in physical activity, and less "screen time". ${ }^{16}$ The pilot program we describe delivered in Indian colleges has potential to have a similar impact if expanded with follow-up education, feedback, and confirmatory testing. In addition, the short educational video that was made YouTube accessible promoted reinforcement outside of the one-time session and could support secondary gains if students share it at home with friends and family. However, it is difficult to determine formally in this study whether the video had any impact at all on the health education of the youth.

The results of our study also highlight the substantial prevalence of risk factors for CKD that are already present in young adults including elevated blood pressure, glycosuria, obesity, and proteinuria. High blood pressure was detected in 5-6\% of the youth screened, depending on whether we used systolic or diastolic cutoffs. Arguably, a diagnosis of sustained hypertension should be based on several readings. However, the methods used to measure and report blood pressure were consistent with prior research ${ }^{17}$ and, given the young age of the participants, are of particular concern because we would not expect high values to begin with.

Using culturally appropriate definitions, remarkably $36 \%$ of our study population was overweight or obese. This rivals numbers seen in many developed countries, which harbor the highest rates of obesity across the world. ${ }^{18}$ Prior work in Northern India shows similar alarming trends in adults. ${ }^{19}$ Whether in adults or youth, these numbers show there is a significant potential for negative impact on kidney health, because a link between obesity and renal dysfunction is well established. ${ }^{20,21}$ Many factors may contribute to increasing prevalence of obesity, including a westernization of diet and a sedentary lifestyle. ${ }^{22}$ Results from this program suggest that there is an important opportunity for early public health intervention toward healthy eating and increasing activity.

Our screened population had a $6 \%$ prevalence of proteinuria. Testing on a urine sample, which is not a first morning sample (as in this study), does bring up questions of false-positive and -negative testing. There is also the potential of transient proteinuria on a one-time dipstick. Nonetheless, the significance of this finding cannot be overstated. Dipstick-positive proteinuria signifies renal disease, which can progress to advanced stages of kidney disease without early identification and medical intervention. The association we found between older age and proteinuria is in line with other observations, ${ }^{8}$ supporting the validity of our results. Contrary to prior research that shows women as having higher odds of proteinuria however, our study found a trend of higher odds in men. We speculate that this could be due to the different demographics in populations (adults 
versus youth) or different comorbidity characteristics that we did not capture, yet to be elucidated in future work. One hundred and thirty-six participants $(6 \%)$ had glycosuria on urine testing. Unfortunately, blood sugar was not tested in the study. Thus, while it is difficult to interpret the significance of the glycosuria, given the high prevalence of overweight and obese students, it is possible that at least some of these could potentially be a marker of underlying glucose intolerance or frank diabetes.

There are limitations of this pilot program. First, it was a community-led effort and not designed for research purposes per se. However, the methods used are comparable to formalized efforts in the past, ${ }^{5}$ and the findings should be shared in order to shed light on the alarming, but potentially modifiable trends of CKD in youth. While it is possible that with more formal testing, such as ambulatory pressure monitoring, the blood pressure of our participants may have changed, the measurements were done to support screening and were performed with standardization to minimize erroneous readings on-site. Another limitation is with urine dipstick measurements that were not early AM sample and were one time; proteinuria may be transient due to various reasons. However, armed with knowledge that feasibility of screening with education is possible, future efforts could and should incorporate subsequent confirmatory testing with follow-up if needed. The lack of measurement of serum creatinine and blood glucose is another limitation; however, this was a grass roots effort at screening and as such screening creatinine and glucose was not part of the plan. Another limitation is the lack of quantification of the impact of the video on screening participation by comparison to a control group; moreover, there was no knowledge assessment after the program, so we have no way of knowing how the education video impacted participant knowledge or was retained over time. This is an area for formal research in a future study. Finally, the convenience, cross-sectional population does not represent all youth within India, especially those of lower socioeconomic status or nonurban populations. We feel that this makes our findings even more relevant because individuals in resource-poor areas are more likely to have an even higher prevalence of risks. ${ }^{23}$

There are still many important implications of this program. Overall, the program was successful and provided education that was disseminated to a large number of young adults within a short time frame and in a proactive manner. While prior research reports on screening for CKD and risk factors in adults, our research provides sobering insight into the current and trending health status evolving in Indian youth. Given the large population and the demographic distribution, the number of young people who could potentially develop CKD with age could run into about 24 million. Our pilot program also offers hope to change these trends. Even a short education session and video appealed to the millennial generation as indicated by the very enthusiastic participation of youth in subsequent urine screening. With advancing technology and social networking, health education could be spread "virally" not only among younger adults but also possibly later via secondary mechanisms (e.g., verbal) to older family members as well. This pilot program balanced efficiency and cost-effectiveness while maximizing use of existing cultural and community resources - supporting feasibility for expansion in the future.

\section{Conclusion}

We offer the current program as a model for screening and educating youth for CKD risk factors. Early detection of CKD is important, and our novel project targeting young adults shows reason that we must begin this process early. There exists a unique opportunity to identify and intervene on several aspects related to nonoptimal health. Furthermore, our pilot program suggests alarming health trends in the Indian population, demanding that more attention be paid to primary health interventions to abate the potential epidemic of CKD.

\section{Acknowledgments}

A portion of the data presented in this manuscript was presented as an oral presentation at the American Society of Nephrology Conference, November 2014, Philadelphia, PA, USA. This work was supported by an NIH grant (NIDDK K23 DK 097183) to JWN. This research and the opinions expressed are the authors and do not reflect those of the funding agency. The study sponsor had no role in the study design, data collection or analysis, interpretation, writing, or submission of the manuscript.

\section{Author contributions}

RR contributed to initial study, program design and implementation and data gathering. JAWN and BWG contributed to data interpretation and analysis. PSR, JAWN, and RLP contributed to important intellectual contributions All authors contributed toward data analysis, drafting and critically revising the paper, gave final approval of the version to be published, and agree to be accountable for all aspects of the work.

\section{Disclosure}

The authors report no conflicts of interest in this work. 


\section{References}

1. Wild S, Roglic G, Green A, Sicree R, King H. Global prevalence of diabetes: estimates for the year 2000 and projections for 2030. Diabetes Care. 2004;27(5):1047-1053.

2. Gupta R. Trends in hypertension epidemiology in India. J Hum Hypertens. 2004;18(2):73-78.

3. Foley RN, Collins AJ. End-stage renal disease in the United States: an update from the United States Renal Data System. J Am Soc Nephrol. 2007;18(10):2644-2648.

4. Powe NR, Boulware LE. Population-based screening for CKD. Am J Kidney Dis. 2009;53(3 suppl 3):S64-S70.

5. Kurella Tamura M, Li S, Chen SC, et al. Educational programs improve the preparation for dialysis and survival of patients with chronic kidney disease. Kidney Int. 2014;85(3):686-692.

6. Mani MK. Prevention of chronic renal failure at the community level. Kidney Int Suppl. 2003;(83):S86-S89.

7. Agarwal SK, Dash SC, Irshad M, Raju S, Singh R, Pandey RM. Prevalence of chronic renal failure in adults in Delhi, India. Nephrol Dial Transplant. 2005;20(8):1638-1642.

8. Singh AK, Farag YM, Mittal BV, et al. Epidemiology and risk factors of chronic kidney disease in India - results from the SEEK (screening and early evaluation of kidney disease) study. BMC Nephrol. 2013;14:114.

9. Ebbeling CB, Pawlak DB, Ludwig DS. Childhood obesity: public-health crisis, common sense cure. Lancet. 2002;360(9331):473-482.

10. Misra A, Chowbey P, Makkar BM, et al; Concensus Group. Consensus statement for diagnosis of obesity, abdominal obesity and the metabolic syndrome for Asian Indians and recommendations for physical activity, medical and surgical management. $J$ Assoc Physicians India. 2009;57:163-170.

11. Narva AS, Briggs M. The National Kidney Disease Education Program: improving understanding, detection, and management of CKD. Am J Kidney Dis. 2009;53(3 supp1 3):S115-S120.
12. Hostetter TH, Lising M. National kidney disease education program. J Am Soc Nephrol. 2003;14(7 suppl 2):S114-S116.

13. Brieger WR, Delano GE, Lane CG, Oladepo O, Oyediran KA. West African youth initiative: outcome of a reproductive health education program. J Adolesc Health. 2001;29(6):436-446.

14. Gortmaker SL, Peterson K, Wiecha J, et al. Reducing obesity via a school-based interdisciplinary intervention among youth: planet health. Arch Pediatr Adolesc Med. 1999;153(4):409-418.

15. Hawkins J, Catalano RF, Kosterman R, Abbott R, Hill KG. Preventing adolescent health-risk behaviors by strengthening protection during childhood. Arch Pediatr Adolesc Med. 1999;153(3):226-234.

16. Eagle TF, Gurm R, Smith CA, et al. A middle school intervention to improve health behaviors and reduce cardiac risk factors. Am J Med. 2013;126(10):903-908.

17. Anupama YJ, Uma G. Prevalence of chronic kidney disease among adults in a rural community in South India: results from the kidney disease screening (KIDS) project. Indian J Nephrol. 2014;24(4):214-221.

18. Ogden CL, Carroll MD, Kit BK, Flegal KM. Prevalence of childhood and adult obesity in the United States, 2011-2012. JAMA. 2014;311(8):806-814.

19. Pengpid S, Peltzer K. Prevalence of overweight/obesity and central obesity and its associated factors among a sample of university students in India. Obes Res Clin Pract. 2014;8(6):e558-e570.

20. Billings FT 4th, Pretorius M, Schildcrout JS, et al. Obesity and oxidative stress predict AKI after cardiac surgery. J Am Soc Nephrol. 2012; 23(7):1221-1228

21. Stenvinkel P, Zoccali C, Ikizler TA. Obesity in CKD - what should nephrologists know? J Am Soc Nephrol. 2013;24(11):1727-1736.

22. Rohilla R, Rajput M, Rohilla J, Malik M, Garg D, Verma M. Prevalence and correlates of overweight/obesity among adolescents in an urban city of north India. J Family Med Prim Care. 2014;3(4):404-408.

23. Rogers R, Eagle TF, Sheetz A, et al. The relationship between childhood obesity, low socioeconomic status, and race/ethnicity: lessons from Massachusetts. Child Obes. 2015;11(6):691-695.

\section{Publish your work in this journal}

The International Journal of Nephrology and Renovascular Disease is an international, peer-reviewed open access journal focusing on the pathophysiology of the kidney and vascular supply. Epidemiology, screening, diagnosis, and treatment interventions are covered as well as basic science, biochemical and immunological studies. The manuscript management system is completely online and includes a very quick and fair peer-review system, which is all easy to use. Visit http://www. dovepress.com/testimonials.php to read real quotes from published authors. 